\title{
Index rerum vol. 13
}

Absorption of iron from food, 44, 207 Absorption of xylose, 370 Alkaline phosphatase and osteomalacia, 153 Anaemia and iron, 1, 2, 12, 31, 44, 52, 61,

207, 246, 296 Aphasia and the word, 307 Audiometric studies, 350 Automation and work (B), 256

Bone marrow and iron, 296

Brain disease, 97, 307

Brain syndrome and cinnarzine, 89

Calcium and osteomalacia, 153 Carcinoid syndrome, 368 Carotene in serum, 370

Catecholamines and excretion, 257 Central disorders of the special senses, 329 Cerebral dysfunction, 97, 181, 192, 307 Central nervous system and degenerative

disorders (B), 96 Cerebral vascular disease, 181, 192, 339 Cholesterol and heart disease, 82

Cholesterol and triglyceride, 171 Cinnarzine and brain syndrome, 89 Collagen metabolism and hydroxyproline,

277 Cornea, 321

Corticotrophin in serum, 215 Cortisol and corticotrophin, 215 Creatinine excretion, 277

Day hospital (B), 320

Deafness, 350

Degenerative disorders of the CNS (B), 96

Dementia and depression, 233, 339 Dental care, 227, 359 Depression and dementia, 233 Digitalis glycosides, 119, 285 Diuretics and side effects, 261 Drug trial, pemoline, 269

Electroencephalogram and brain disease,

97 Epithelial changes, 61 Electrocardiogram, sodium and potassium,

233 Epidemiology and iron deficiency, 2 Extracellular fluid, sodium and potassium, 233 Eye, 321

Femoral neck and osteomalacia, 153 Foster-Moore's syndrome, 321

Gastrointestinal tract and iron, 207

Haemoglobin, 12, 52, 61, 227, 246, 296 Heart contractility and digitalis glycosides, 285 Heart disease and ischaemia, 82 Hospital management, 136, 161 Hydroxyproline and collagen metabolism,

277 Hydroxyproline-creatinine ratio, 277 Hypokalaemia, 114, 119

Iron and anaemia, 1, 2, 12, 31, 44, 52, 61,

207, 246, 296 Ischaemic heart disease, 82

380

Index rerum vol. 13

Jaundice, 69

Lung function and smoking, 125 Lung, mechanical properties (B), 96 Lipid changes in overweight, 171 Liver biopsy and jaundice, 69

Macula, 321 
Mastication and nutrition, 227 Megaloblastic anaemia, 12 Muscle strength and potassium, 221 Neurology, 97, 339 Nursing care, 161, 136 Nutritional deficiencies, 227 Nyctohemeral rhythm of corticotrophin, 215

Oral problems, 359 Osteomalacia, 153

Pemoline, drug trial, 269 Potassium, depression and dementia, 233 Potassium intake, 114, 119, 221 Potassium and muscle strength, 221 Physical illness in social emergencies, 145,

192 Phosphorus and osteomalacia, 153 Presbycusis, 350

Rehabilitation, 181, 192

Renin activity, depression and dementia, 233 Respiratory failure and smoking, 125 Retina, 321

Senses, central disorders, 329

Sensory deprivation, 97

Serum carotene level, 370

Serum corticotrophin, 215

Serum iron content, 12, 52, 246, 296

Serum lipids and overweight, 171

Sodium, depression and dementia, 233

Sodium, tubular reabsorption, 261

Social emergencies, 145

Strokes and vascular disease, 181

Sympathetico-adrenal system, 257

Teeth, 227, 359

Tissue changes and iron, 61

Toxicity of digitalis glycosides, 285

Transferrin and iron deficiency, 31, 207, 296

Triglyceride and cholesterol, 171

Urinary excretion of catecholamines, 257

Word and aphasia, 307

Vascular disease of the brain, 181, 192, 339 Vitamin B12, 12, 227, 296, 370

Xylose absorption, 370 